\title{
sciendo
}

RESEARCH PAPERS FACULTY OF MATERIALS

SCIENCE AND TECHNOLOGY IN TRNAVA

SLOVAK UNIVERSITY OF TECHNOLOGY

IN BRATISLAVA

2021, Volume 29, Number 49

DOI 10.2478/rput-2021-0027

\section{A TWO WHEEL SELF-BALANCING VEHICLE}

\author{
Rastislav ĎURIŠ ${ }^{1}$, Eva LABAŠOVÁ ${ }^{1}$, Jarmila ORAVCOVÁ ${ }^{1}$, Štefan ŠIMON ${ }^{1}$ \\ ${ }^{1}$ Slovak University of TeChNOLOGy in BRAtislava, \\ FACULTY OF MATERIALS SCIENCE AND TECHNOLOGY IN TRNAVA, \\ INSTITUTE OF APPLIED INFORMATICS, AUTOMATION AND MECHATRONICS, \\ Ulica JÁna Bottu 2781/25, 91724 TRNAVA, SlOVAK REPUBliC \\ e-mail: rastislav.duris@stuba.sk, eva.labasova@stuba.sk,jarmila.oravcova@stuba.sk, \\ stefan.simon@stuba.sk \\ Received 26 August 2021, Accepted 28 October 2021, Published 24 November 2021
}

\begin{abstract}
This paper deals with the development of the equation of motion and practical implementation of low cost two-wheel self-balancing model of a Segway transporter. The experimental model of cart was designed and made under this study. Nonlinear equations of motion of real model and linearized model were derived. To develop the mathematical model, Matlab/Simulink was applied. The mechanical part was implemented into Simulink, and a DC motor was considered as a linear system. The real model was tested for its balance by implementation of a control algorithm consisting of a complementary filter and PID algorithm on an Arduino development board with peripheral devices. The fully functional self-balancing model was used as a demonstration in the teaching process of the Mechatronics courses.
\end{abstract}

\section{Keywords}

Segway model, equations of motion, self-balancing, PID control

\section{INTRODUCTION}

A two-wheel self-balancing cart uses the idea of an inverted pendulum theory, which is a conventional non-linear control problem of unstable system. Many publications deal with the problem of inverted pendulum, using different approaches to solution: starting from simple balancing of a two-wheel transporter (only 2-DOF) [8], to complex decomposed systems. Some papers are focused on description or comparison of balancing control techniques (PID, LQR, state space model, transfer function, fuzzy control, neural networks) $[2,3,4,9,10]$, or on filtering the measured signals $[1,6,7]$.

This paper is aimed at designing a simple autonomous model of a "Segway" transportation system, which can be used to demonstrate principles of stability control in the Mechatronics lectures. That leads to several simplifications introduced into the physical and mathematical models. To balance the two-wheel cart, it is necessary to obtain accurate information of the actual 
tilt angle by using an Inertial Measurement Unit. Output data from IMU are combined in a complementary filter. The vehicle utilises a PID controller to control its stability. Advantage of PID control is a simple set up to a wide application range and undemanding implementation.

\section{MATHEMATICAL MODEL OF A TWO-WHEEL CART}

Mathematical description of a two-wheel vehicle by differential equations is based on a simplified physical model. The following assumptions and simplifications were used for the problem:

- the friction between all components is neglected,

- the efficiency of the gearbox is equal to 1 , and the reduction is rigid,

- rolling of the wheels on the mat without slipping is considered,

- only a small inclination angle $\theta$ of the vehicle from the equilibrium position is assumed.

We used a simplified physical model based on the inverted pendulum to assemble the mathematical description and construct the dynamic equations describing the movement of the vehicle. Fig. 1 shows the simplified model of the two-wheel self-balancing cart. Subscript $B$ denotes parameters and dimensions of chassis (body), and subscript $W$ denotes wheel parameters.

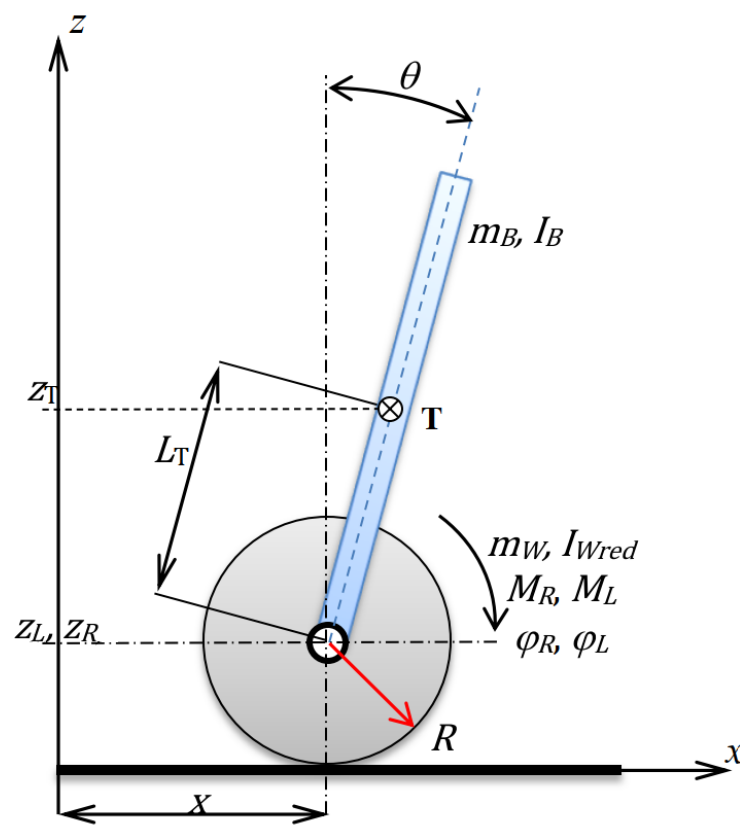

$x$ - horizontal position of the wheels axis

$L_{\mathrm{T}}$ - distance between center of mass and the wheels axis

$I_{B}$ - moment of inertia of the chassis

$I_{W}$ - moment of inertia of the wheel

$I_{\text {Wred }}$ - reduced moment of inertia of the wheel, gear and motor

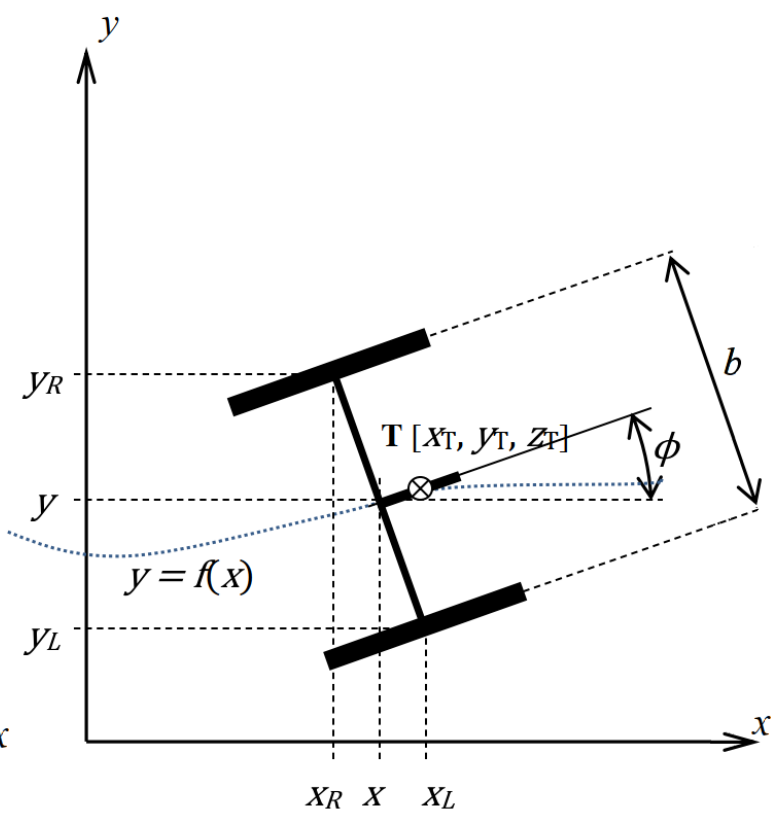

$m_{B}$ - weight of the chassis $m_{W}-$ weight of the wheel $\varphi_{\mathrm{R}}, \varphi_{\mathrm{L}}-$ angles of the wheels $\theta$ - tilt angle of the chassis $\phi$ - pan angle of the car $M_{R}, M_{L}-$ driving torques of both DC motors $R$ - radius of the wheel $b$ - wheels distance

Fig. 1 Kinematic model of two-wheel self-balancing cart during general motion in a plane

Derivation of equations of motions was divided into two parts. In the first step, rectilinear in-plane movement of the vehicle was considered. Obtained nonlinear equations of rectilinear motion were linearized and prepared for the assembly of a control model. In the next step, equations of general in-plane motion were derived. 


\section{The equations of motions of two wheel car during rectilinear movement}

When the car moves along a straight line, the coordinates of the centre of gravity $\mathrm{T}$ of the chassis part are defined as

$$
\begin{aligned}
& x_{\mathrm{T}}=x+L_{\mathrm{T}} \sin \theta \\
& y_{\mathrm{T}}=R+L_{\mathrm{T}} \cos \theta
\end{aligned},
$$

where $x=\varphi R(\dot{x}=\dot{\varphi} R)$, whereas $\varphi=\varphi_{L}=\varphi_{R}$. The position of the centre of mass of chassis and all mass parameters need to be determined experimentally. The kinetic energy of the system is equal to

$$
\begin{aligned}
E_{k} & =\frac{1}{2} m_{B} \dot{x}_{\mathrm{T}}^{2}+\frac{1}{2} I_{B} \dot{\theta}^{2}+2\left[\frac{1}{2} m_{W} \dot{x}^{2}+\frac{1}{2} I_{W} \dot{\varphi}^{2}\right]= \\
& =\left(\frac{1}{2} m_{B}+m_{W}+\frac{I_{W}}{R^{2}}\right) \dot{x}_{\mathrm{T}}^{2}+m_{B} L_{\mathrm{T}} \cos \theta \dot{\theta} \dot{x}+\frac{1}{2}\left(m_{B} L_{\mathrm{T}}^{2}(\cos \theta)^{2}+I_{B}\right) \dot{\theta}^{2}
\end{aligned} .
$$

The potential energy is given by the vertical position of the chassis center of mass

$$
E_{p}=m_{B} L_{\mathrm{T}} g \cos \theta \text {. }
$$

Given the previous, it is possible to give the expression of the Lagrangian, for the system is written as the expression

$$
L=E_{k}-E_{p}
$$

Having this, the Lagrange's equations become

$$
\begin{aligned}
& \frac{\partial}{\partial t}\left(\frac{\partial L}{\partial \dot{\theta}}\right)-\frac{\partial L}{\partial \theta}=-M \\
& \frac{\partial}{\partial t}\left(\frac{\partial L}{\partial \dot{x}}\right)-\frac{\partial L}{\partial x}=0
\end{aligned}
$$

After some manipulations, we obtain motion equations of inverted pendulum model

$$
\begin{aligned}
& m_{B} L_{\mathrm{T}} \cos \theta \ddot{x}-m_{B} L_{\mathrm{T}}^{2} \cos \theta \sin \theta \dot{\theta}^{2}+\left(m_{B} L_{\mathrm{T}}^{2}(\cos \theta)^{2}+I_{C}\right) \ddot{\theta}-m_{B} L_{\mathrm{T}} g \sin \theta=-M \\
& \left(m_{B}+2 m_{W}+\frac{2 I_{W}}{R^{2}}\right) \ddot{x}-m_{B} L_{\mathrm{T}} \sin \theta \dot{\theta}^{2}+m_{B} L_{\mathrm{T}} \cos \theta \ddot{\theta}=0
\end{aligned}
$$

where $M$ represents the required torque of DC motor with gear box.

Solution of the system of nonlinear differential equations was accomplished in MATLAB/Simulink. The model is shown in Fig. 2. 


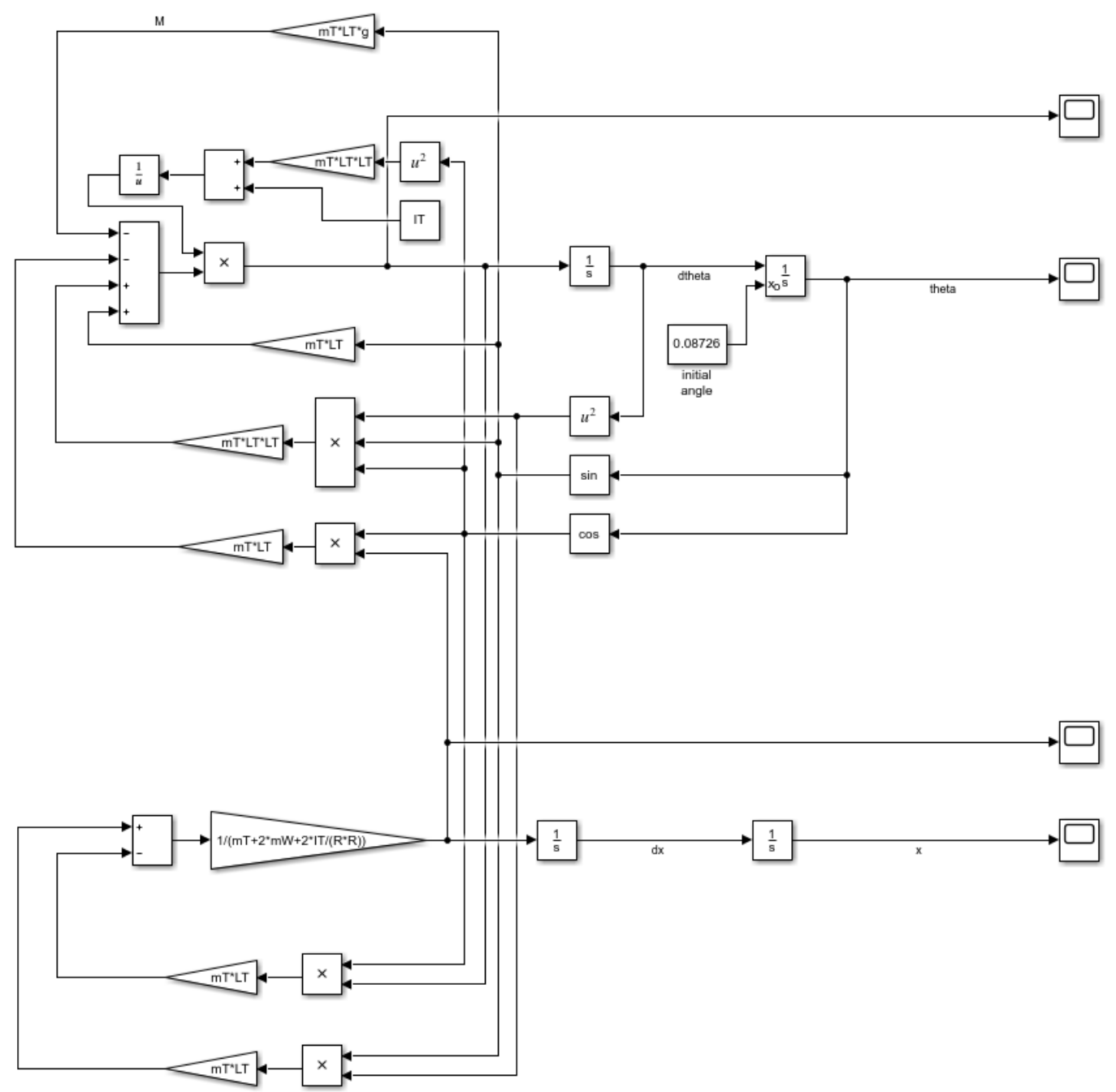

Fig. 2 Model of self-balance vehicle in MATLAB/Simulink

Variation of the inclination angle $\theta$ and horizontal position of the axis of wheel $x$ in dependence on time as a solution of non-linear model in Fig. 2 without implementation of PID in feedback is shown in Fig. 3.

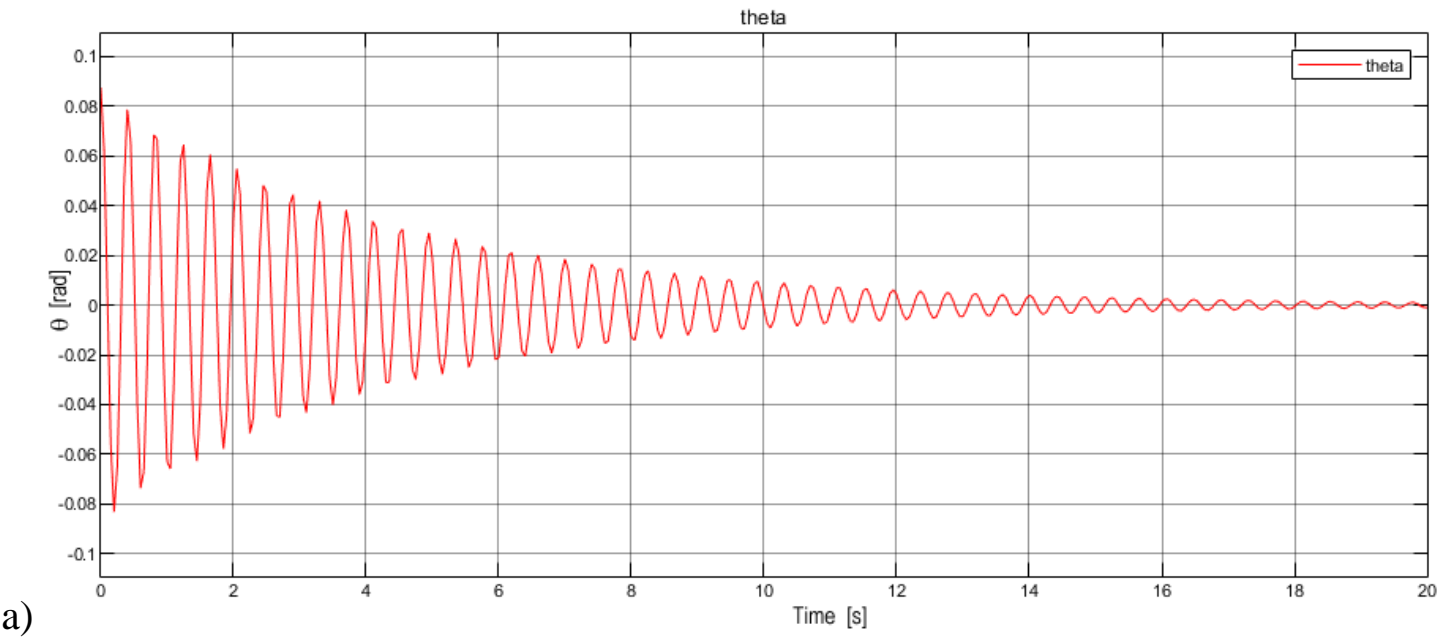


b)

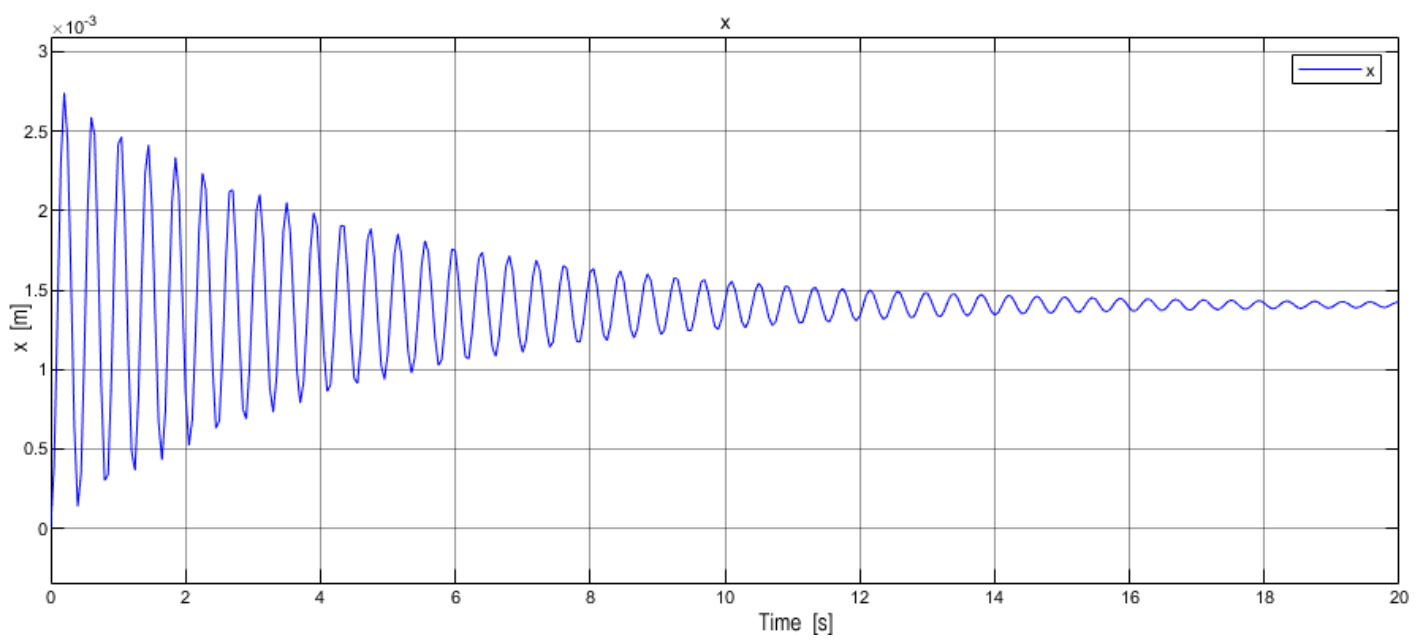

Fig. 3 Response of tilt angle $\theta$ and position $x$ of the wheel axis

The linearization of the system of equations Eqs. 6 is performed for a small tilt angle $\left(\theta<5^{\circ}\right)$, and we can introduce

$$
\sin \theta \approx \theta \quad \cos \theta \approx 1 \quad \dot{\theta}^{2} \approx 0 .
$$

In consequence of these, from linearized form of Eqs. 6, translational and angular acceleration of the car could be calculated as

$$
\begin{gathered}
\ddot{x}=\frac{1}{\left[m_{T}^{2} L_{T}^{2}-k\left(m_{T} L_{T}^{2}+I_{T}\right)\right]}+m_{T}^{2} L_{T}^{2} g \theta-m_{T} L_{T} M \\
\ddot{\theta}=\frac{1}{\left[m_{T}^{2} L_{T}^{2}-k\left(m_{T} L_{T}^{2}+I_{T}\right)\right]}+k m_{T} L_{T} g \theta-k M,
\end{gathered}
$$

where $k=m_{B}+2 m_{W}+\frac{2 I_{W}}{R^{2}}$.

\section{The equations of motions of a two-wheel car during general in-plane movement}

The general case of moving a two-wheeled vehicle is moving along a curved path in a plane. If the path of movement is written as the function $f(x)$, the pan angle $\phi$ of rotation of the vehicle about the vertical axis is given by the relation

$$
\phi=\operatorname{arctg} \frac{d f(x)}{d x} .
$$

In the case of rectilinear motion, the motion of the vehicle can be described by the equations derived in the previous section. When the direction of the vehicle movement changes, we introduce the assumption that the trajectories of significant points on the body of the vehicle are parts of circles as shown in Fig. 4. 


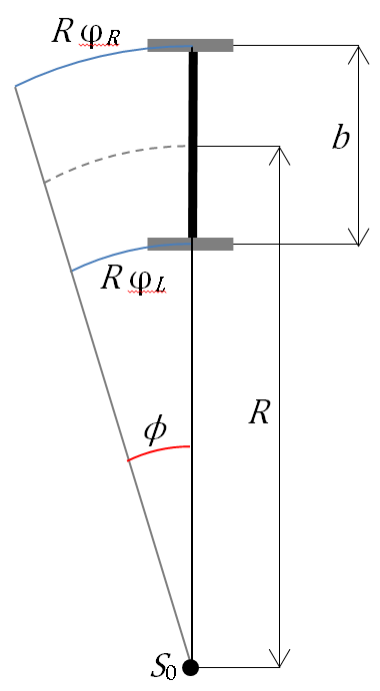

Then, for the radius of curvature of the track $R$ in the middle of the vehicle axis, it applies

$$
R=\frac{b}{2} \frac{\varphi_{R}+\varphi_{L}}{\varphi_{R}-\varphi_{L}} .
$$

The angle of rotation of the vehicle $\phi$ about the vertical axis can be determined from the difference between the travel paths of the right and left wheels caused by different wheel speeds

$$
\operatorname{tg} \phi=\frac{R}{b}\left(\varphi_{R}-\varphi_{L}\right) .
$$

Fig. 4 Movement of vehicle wheels along a part of a circular path

Changes of the coordinates of significant points on the vehicle in accordance with Fig. 1 as a function of time and their time derivatives are summarized in the following Table 1.

\begin{tabular}{|l|l|}
\hline \multicolumn{2}{|l|}{ Table 1 Coordinates of significant points on car and their derivatives } \\
\hline$x_{\mathrm{T}}=x+L_{\mathrm{T}} \sin \theta \cos \phi$ & $\dot{x}_{\mathrm{T}}=\dot{x}+L_{\mathrm{T}}(\dot{\theta} \cos \theta \cos \phi-\dot{\phi} \sin \theta \sin \phi)$ \\
$y_{\mathrm{T}}=y+L_{\mathrm{T}} \sin \theta \sin \phi$ & $\dot{y}_{\mathrm{T}}=\dot{y}+L_{\mathrm{T}}(\dot{\theta} \cos \theta \sin \phi+\dot{\phi} \sin \theta \cos \phi)$ \\
$z_{\mathrm{T}}=R_{w}+L_{\mathrm{T}} \cos \theta$ & $\dot{z}_{\mathrm{T}}=-L_{\mathrm{T}} \dot{\theta} \sin \theta$ \\
& $\ddot{x}_{\mathrm{T}}=\ddot{x}+L_{\mathrm{T}}\left(\ddot{\theta} \cos \theta \cos \phi-\dot{\theta}^{2} \sin \theta \cos \phi-\right.$ \\
& $\left.2 \dot{\theta} \dot{\phi} \cos \theta \sin \phi-\quad \ddot{\phi} \sin \theta \sin \phi-\dot{\phi}^{2} \sin \theta \cos \phi\right)$ \\
& $\ddot{y}_{\mathrm{T}}=\ddot{y}+L_{\mathrm{T}}\left(\ddot{\theta} \cos \theta \sin \phi-\dot{\theta}^{2} \sin \theta \sin \phi+2 \dot{\theta} \dot{\phi} \cos \theta \cos \phi\right.$ \\
& $\ddot{z}_{\mathrm{T}}=-L_{\mathrm{T}}\left(\ddot{\theta} \sin \theta+\dot{\theta}^{2} \cos \theta\right)$ \\
\hline$x_{L}=x+\frac{1}{2} b \sin \phi$ & $\left.\dot{x}_{L}=\dot{x}+\frac{1}{2} b \dot{\phi} \cos \phi \sin \phi\right)$ \\
$y_{L}=y-\frac{1}{2} b \cos \phi$ & $\dot{y}_{L}=\dot{y}+\frac{1}{2} b \dot{\phi} \sin \phi$ \\
$z_{L}=R_{w}$ & $\ddot{x}_{L}=\ddot{x}+\frac{1}{2} b\left(\ddot{\phi} \cos \phi-\dot{\phi}^{2} \sin \phi\right)$ \\
& $\ddot{y}_{L}=\ddot{y}+\frac{1}{2} b\left(\ddot{\phi} \sin \phi+\dot{\phi}^{2} \cos \phi\right)$ \\
& \\
\hline$x_{R}=x-\frac{1}{2} b \sin \phi$ & $\dot{x}_{R}=\dot{x}-\frac{1}{2} b \dot{\phi} \cos \phi$ \\
$y_{R}=y+\frac{1}{2} b \cos \phi$ & $\dot{y}_{R}=\dot{y}-\frac{1}{2} b \dot{\phi} \sin \phi$ \\
$z_{R}=R_{w}$ & $\ddot{x}_{R}=\ddot{x}-\frac{1}{2} b\left(\ddot{\phi} \cos \phi-\dot{\phi}^{2} \sin \phi\right)$ \\
& $\ddot{y}_{R}=\ddot{y}-\frac{1}{2} b\left(\ddot{\phi} \sin \phi+\dot{\phi}^{2} \cos \phi\right)$ \\
& \\
&
\end{tabular}




$$
\begin{array}{|l|l|}
\hline \phi=\operatorname{arctg} \frac{R}{b}\left(\varphi_{R}-\varphi_{L}\right) & \dot{\phi}=\frac{R\left(\dot{\varphi}_{R}-\dot{\varphi}_{L}\right)}{b\left(1+\frac{R^{2}}{b^{2}}\left(\varphi_{R}-\varphi_{L}\right)^{2}\right)} \\
& \ddot{\phi}=\frac{R\left(\ddot{\varphi}_{R}-\ddot{\varphi}_{L}\right)}{b+\frac{R^{2}}{b}\left(\varphi_{R}-\varphi_{L}\right)^{2}}-\frac{2 R^{3}\left(\dot{\varphi}_{R}-\dot{\varphi}_{L}\right)^{2}\left(\varphi_{R}-\varphi_{L}\right)}{b^{3}\left(1+\frac{R^{2}}{b^{2}}\left(\varphi_{R}-\varphi_{L}\right)^{2}\right)^{2}} \\
\hline
\end{array}
$$

When the car moves along a curved path, it is necessary to include in the solution the kinetic energy from the rotation of the vehicle about the vertical axis. The equations of motion are derived using Lagrange's equations and the kinetic and potential energy of the system

$$
\begin{aligned}
& \frac{d}{d t}\left(\frac{\partial L}{\partial \dot{\theta}}\right)-\frac{\partial L}{\partial \theta}=-n\left(M_{R}+M_{L}\right) \\
& \frac{d}{d t}\left(\frac{\partial L}{\partial \dot{\varphi}_{R}}\right)-\frac{\partial L}{\partial \varphi_{R}}=n M_{R} \\
& \frac{d}{d t}\left(\frac{\partial L}{\partial \dot{\varphi}_{L}}\right)-\frac{\partial L}{\partial \varphi_{L}}=n M_{L} .
\end{aligned}
$$

The total kinetic energy of the vehicle consists of the kinetic energy of the superstructure and both wheels in the general shape

$$
E_{k}=\frac{1}{2} m_{B} \bar{v}_{\mathrm{T}}^{2}+\frac{1}{2} I_{B} \dot{\theta}^{2}+\frac{1}{2} m_{W}\left(\bar{v}_{R}^{2}+\bar{v}_{L}^{2}\right)+\frac{1}{2} I_{\text {Wred }}\left(\dot{\varphi}_{R}^{2}+\dot{\varphi}_{L}^{2}\right)+\frac{1}{2} I_{V} \dot{\phi}^{2} .
$$

When the vehicle moves along in-plane path, the total kinetic energy $E_{k}$ of the system is expressed by the relation

$$
\begin{aligned}
& E_{k}=\frac{1}{2} m_{B}\left(\dot{x}_{\mathrm{T}}^{2}+\dot{y}_{\mathrm{T}}^{2}\right)+\frac{1}{2} I_{B} \dot{\theta}^{2}+\frac{1}{2} m_{W}\left(\dot{x}_{R}^{2}+\dot{y}_{R}^{2}\right)+\frac{1}{2} m_{W}\left(\dot{x}_{L}^{2}+\dot{y}_{L}^{2}\right) \\
& +\frac{1}{2} I_{W r e d}\left(\dot{\varphi}_{R}^{2}+\dot{\varphi}_{L}^{2}\right)+\frac{1}{2} I_{V} \dot{\phi}^{2}= \\
& =\frac{1}{2}\left[m_{B}\left(\dot{x}_{\mathrm{T}}^{2}+\dot{y}_{\mathrm{T}}^{2}\right)+I_{B} \dot{\theta}^{2}+m_{W}\left(\dot{x}_{R}^{2}+\dot{y}_{R}^{2}+\dot{x}_{L}^{2}+\dot{y}_{L}^{2}\right)+I_{\text {Wred }}\left(\dot{\varphi}_{R}^{2}+\dot{\varphi}_{L}^{2}\right)+I_{V} \dot{\phi}^{2}\right]
\end{aligned}
$$

The total potential energy $E_{p}$ of the system is equal to the potential energy of the chassis only, which is given by Eq. 3. The masses, moments of inertia of various parts of the vehicle and the position of the centre of gravity must be determined experimentally. The Lagrangian $L$ takes the form

$$
\begin{aligned}
& L=E_{k}-E_{p}= \\
& \quad=\frac{1}{2} m_{B}\left(\dot{x}_{\mathrm{T}}^{2}+\dot{y}_{\mathrm{T}}^{2}\right)+\frac{1}{2} I_{B} \dot{\theta}^{2}+\frac{1}{2} m_{W}\left(\dot{x}_{R}^{2}+\dot{y}_{R}^{2}+\dot{x}_{L}^{2}+\dot{y}_{L}^{2}\right)+\frac{1}{2} I_{W r e d}\left(\dot{\varphi}_{R}^{2}+\dot{\varphi}_{L}^{2}\right)+ \\
& +\frac{1}{2} I_{V} \dot{\phi}^{2}-m_{B} g L_{\mathrm{T}} \cos \theta
\end{aligned}
$$

After substituting into Lagrange's equations and necessary manipulations, we obtain nonlinear differential equations describing the motion of parts of a two-wheel vehicle with the following derivatives

$$
\begin{aligned}
& \frac{d}{d t}\left(\frac{\partial L}{\partial \dot{\theta}}\right)=m_{B} L_{T}\left(\ddot{x_{T}} \cos \theta \cos \phi-\dot{\theta} \dot{x_{T}} \sin \theta \cos \phi-\dot{\phi} \dot{x_{T}} \cos \theta \sin \phi+\ddot{y_{T}} \cos \theta \sin \phi-\right. \\
& \left.\dot{\theta} \dot{y}_{T} \sin \theta \cos \phi+\dot{\phi} y_{T} \cos \theta \cos \phi\right)+I_{B} \ddot{\theta}
\end{aligned}
$$




$$
\begin{gathered}
\frac{d}{d t}\left(\frac{\partial \dot{\phi}}{\partial \dot{\varphi}_{R}}\right)=-\frac{8 R^{4}\left(\varphi_{R}-\varphi_{L}\right)\left(\dot{\varphi}_{R}-\dot{\varphi}_{L}\right)^{2}}{b^{4}\left(1+\frac{R^{2}}{b^{2}}\left(\varphi_{R}-\varphi_{L}\right)^{2}\right)^{3}}+\frac{2 R^{2}\left(\ddot{\varphi}_{R}-\ddot{\varphi}_{L}\right)}{b^{2}\left(1+\frac{R^{2}}{b^{2}}\left(\varphi_{R}-\varphi_{L}\right)^{2}\right)^{2}} \\
\frac{d}{d t}\left(\frac{\partial \dot{\phi}}{\partial \dot{\varphi}_{L}}\right)=\frac{8 R^{4}\left(\varphi_{R}-\varphi_{L}\right)\left(\dot{\varphi}_{R}-\dot{\varphi}_{L}\right)^{2}}{b^{4}\left(1+\frac{R^{2}}{b^{2}}\left(\varphi_{R}-\varphi_{L}\right)^{2}\right)^{3}}-\frac{2 R^{2}\left(\ddot{\varphi}_{R}-\ddot{\varphi}_{L}\right)}{b^{2}\left(1+\frac{R^{2}}{b^{2}}\left(\varphi_{R}-\varphi_{L}\right)^{2}\right)^{2}}
\end{gathered}
$$

where

$$
\begin{aligned}
& \frac{\partial L}{\partial \theta}=m_{B}\left(\dot{x_{T}} \frac{\partial x_{T}}{\partial \theta}+\dot{y_{T}} \frac{\partial \dot{y}_{T}}{\partial \dot{\theta}}\right)+\dot{\theta} m_{B} g L_{T} \sin \theta \\
& \frac{\partial \dot{x}_{T}}{\partial \theta}=L_{T}\left(-\dot{\theta}^{2} \sin \theta \cos \phi-\dot{\theta} \dot{\phi} \cos \theta \sin \phi\right) \\
& \frac{\partial \dot{y}_{T}}{\partial \theta}=L_{T}\left(-\dot{\theta^{2}} \sin \theta \sin \phi+\dot{\theta} \dot{\phi} \cos \theta \cos \phi\right) \\
& \frac{\partial L}{\partial \varphi_{R}}=m_{B}\left(\dot{x_{T}} \frac{\partial \dot{x_{T}}}{\partial \varphi_{R}}+\dot{y_{T}} \frac{\partial \dot{y}_{T}}{\partial \varphi_{R}}\right)+m_{W}\left(\dot{x_{R}} \frac{\partial \dot{x}_{R}}{\partial \varphi_{R}}+\dot{y_{R}} \frac{\partial \dot{y}_{R}}{\partial \varphi_{R}}+\dot{x_{L}} \frac{\partial \dot{x_{L}}}{\partial \varphi_{R}}+\dot{y_{L}} \frac{\partial \dot{y}_{L}}{\partial \varphi_{R}}\right)+I_{v} \dot{\phi} \frac{\partial \dot{\phi}}{\partial \varphi_{R}} \\
& \frac{\partial \dot{x}_{T}}{\partial \varphi_{R}}=L_{T} \dot{\theta} \sin \theta(-\sin \phi) \frac{\partial \phi}{\partial \varphi_{R}}-\sin \theta \frac{\partial \dot{\phi}}{\partial \varphi_{R}} \sin \phi-\dot{\phi} \sin \theta \cos \phi \frac{\partial \phi}{\partial \varphi_{R}} \\
& \frac{\partial \phi}{\partial \varphi_{R}}=\frac{R}{b\left(1+\frac{R^{2}}{b^{2}}\left(\varphi_{R}-\varphi_{L}\right)^{2}\right)} \\
& \frac{\partial \dot{\phi}}{\partial \varphi_{R}}=\frac{-2 R^{3}\left(\varphi_{R}-\dot{\varphi}_{L}\right)\left(\varphi_{R}-\varphi_{L}\right)}{b^{3}\left(1+\frac{R^{2}}{b^{2}}\left(\varphi_{R}-\varphi_{L}\right)^{2}\right)^{2}} \\
& \frac{\partial \dot{y}_{T}}{\partial \varphi_{R}}=L_{T}\left(\dot{\theta} \cos \theta \cos \phi \frac{\partial \phi}{\partial \varphi_{R}}+\sin \theta \frac{\partial \dot{\phi}}{\partial \varphi_{R}} \cos \phi-\dot{\phi} \sin \phi \frac{\partial \phi}{\partial \varphi_{R}}\right. \\
& \frac{\partial \dot{x}_{R}}{\partial \varphi_{R}}=-\frac{1}{2} b\left(\frac{\partial \dot{\phi}}{\partial \varphi_{R}} \cos \phi-\dot{\emptyset} \sin \phi \frac{\partial \phi}{\partial \varphi_{R}}\right) \\
& \frac{\partial \dot{y}_{R}}{\partial \varphi_{R}}=-\frac{1}{2} b\left(\frac{\partial \dot{\phi}}{\partial \varphi_{R}} \sin \phi+\dot{\phi} \cos \phi \frac{\partial \phi}{\partial \varphi_{R}}\right) \\
& \frac{\partial \dot{x}_{L}}{\partial \varphi_{R}}=\frac{1}{2} b\left(\frac{\partial \dot{\phi}}{\partial \varphi_{R}} \cos \phi-\dot{\phi} \sin \phi \frac{\partial \phi}{\partial \varphi_{R}}\right) \\
& \frac{\partial \dot{y}_{L}}{\partial \varphi_{R}}=\frac{1}{2} b\left(\frac{\partial \dot{\phi}}{\partial \varphi_{R}} \sin \phi-\dot{\phi} \sin \phi \frac{\partial \phi}{\partial \varphi_{R}}\right) \\
& \frac{\partial L}{\partial \varphi_{L}}=m_{B}\left(\dot{x_{T}} \frac{\partial \dot{x_{T}}}{\partial \varphi_{L}}+\dot{y_{T}} \frac{\partial \dot{y_{T}}}{\partial \varphi_{L}}\right)+m_{W}\left(\dot{x_{R}} \frac{\partial \dot{x}_{R}}{\partial \varphi_{L}}+\dot{y_{R}} \frac{\partial \dot{y}_{R}}{\partial \varphi_{L}}+\dot{x_{L}} \frac{\partial \dot{x_{L}}}{\partial \varphi_{L}}+\dot{y_{L}} \frac{\partial \dot{y}_{L}}{\partial \varphi_{L}}\right)+I_{v} \dot{\phi} \frac{\partial \dot{\phi}}{\partial \varphi_{L}} \\
& \frac{\partial \dot{x}_{T}}{\partial \varphi_{L}}=L_{T} \dot{\theta} \cos \theta(-\sin \phi) \frac{\partial \phi}{\partial \varphi_{L}}-\sin \theta \frac{\partial \dot{\phi}}{\partial \varphi_{L}} \sin \phi-\dot{\phi} \sin \theta \cos \phi \frac{\partial \phi}{\partial \varphi_{L}} \\
& \frac{\partial \phi}{\partial \varphi_{L}}=\frac{-R}{b\left(1+\frac{R^{2}}{b^{2}}\left(\varphi_{R}-\varphi_{L}\right)^{2}\right)} \\
& \frac{\partial \dot{\phi}}{\partial \varphi_{L}}=\frac{2 R^{3}\left(\varphi_{R}-\dot{\varphi}_{L}\right)\left(\varphi_{R}-\varphi_{L}\right)}{b^{3}\left(1+\frac{R^{2}}{b^{2}}\left(\varphi_{R}-\varphi_{L}\right)^{2}\right)^{2}} \\
& \frac{\partial y_{T}}{\partial \varphi_{L}}=L_{T}\left(\dot{\theta} \cos \theta \cos \phi \frac{\partial \phi}{\partial \varphi_{L}}+\sin \theta \frac{\partial \dot{\phi}}{\partial \varphi_{L}} \cos \phi-\dot{\phi} \sin \phi \sin \phi \frac{\partial \phi}{\partial \varphi_{L}}\right. \\
& \frac{\partial \dot{x}_{R}}{\partial \varphi_{L}}=-\frac{1}{2} b\left(\frac{\partial \dot{\phi}}{\partial \varphi_{L}} \cos \phi-\dot{\phi} \sin \phi \frac{\partial \phi}{\partial \varphi_{L}}\right)
\end{aligned}
$$




$$
\begin{aligned}
& \frac{\partial \dot{y}_{R}}{\partial \varphi_{L}}=-\frac{1}{2} b\left(\frac{\partial \dot{\phi}}{\partial \varphi_{L}} \sin \phi+\dot{\phi} \cos \phi \frac{\partial \phi}{\partial \varphi_{L}}\right) \\
& \frac{\partial \dot{x}_{L}}{\partial \varphi_{L}}=\frac{1}{2} b\left(\frac{\partial \dot{\phi}}{\partial \varphi_{L}} \cos \phi-\dot{\phi} \sin \phi \frac{\partial \phi}{\partial \varphi_{L}}\right) \\
& \frac{\partial \dot{y}_{L}}{\partial \varphi_{L}}=\frac{1}{2} b\left(\frac{\partial \dot{\phi}}{\partial \varphi_{L}} \sin \phi-\dot{\phi} \sin \phi \frac{\partial \phi}{\partial \varphi_{L}}\right)
\end{aligned}
$$

In the equations above, the reduced moment of inertia $I_{w r e d}$ takes into account the mass moments of inertia of the rotor of the DC motor, gearbox, shafts and wheels (Fig. 5). The reduction method was used to calculate $I_{\text {wred }}$, while neglecting the moments of inertia of the gears, as the gearbox used the wheels made of plastics. Their mass and moments of inertia are small compared to the mass parameters of other elements in the subassembly. The reduced moment of inertia of the wheel is expressed as

$$
I_{\text {Wred }}=I_{w}+n^{2} I_{m},
$$

where $I_{W}$ is moment of inertia of the wheel, $I_{m}$ is moment of inertia of the DC rotor and $n$ is the gear ratio.

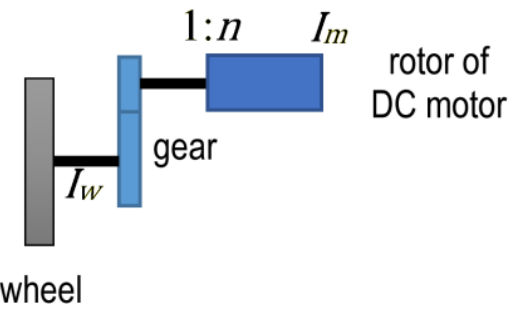

Fig. 5 Wheel drive subsystem

\section{MATHEMATICAL MODEL OF A DC MOTOR}

Mathematical model of a DC motor is often considered in the form of a linear model. Such model is then implemented into the dynamic model of the self-balancing cart to provide a relationship between the applied input voltage $V_{a}$ of DC motor and its output torque $M_{m}$. Torque $M_{m}=k_{m} i$ is proportional to the current $i$. Linear differential equation of the DC motor results from the Kirchhoff's Voltage Law

$$
V_{a}=R i+L \frac{d i}{d t}+V_{e} \quad \Rightarrow \quad V_{a} \approx R i+k_{e} \omega,
$$

where $V_{e}=k_{e} \omega$ is the back electromotive force (voltage) approximated as a linear function of angular velocity. Using the Newton's Law of motion, dynamic equilibrium equation of drive takes the form

$$
I_{R} \frac{d \omega}{d t}=M_{m}-M=k_{m} i-M,
$$

where $I_{R}$ is reduced moment inertia of the drive and $M$ is applied torque. Substituting Eq. 14 into Eq. 15, and simplifying and rearranging the terms leads to the final differential equation which joins motor angular velocity $\omega$, applied voltage $V_{a}$ and applied torque $M$ together 


$$
I_{R} \frac{d \omega}{d t}=-\frac{k_{m} k_{e}}{R} \omega+\frac{1}{R}-M
$$

\section{Static torque characteristics of DC motor}

The dependence of the DC motor torque on the speed can be also determined from the static characteristics. The static gear characteristics of a DC motor with a permanent magnet in the stator can be replaced by a linear dependence according to Fig. 6. The dependence of motor torque $M(\omega)$ is then given by

$$
M(\dot{\varphi})=M_{\text {init }}\left(\frac{1}{\omega_{0}} \dot{\varphi}+1\right)
$$

where $M_{\text {init }}$ is the motor torque at zero speed (usually $\left.M_{\text {init }}=(6 \div 7) M_{n}\right), M_{n}$ is nominal torque of the motor and $\omega_{0}$ is angular speed of unloaded motor.

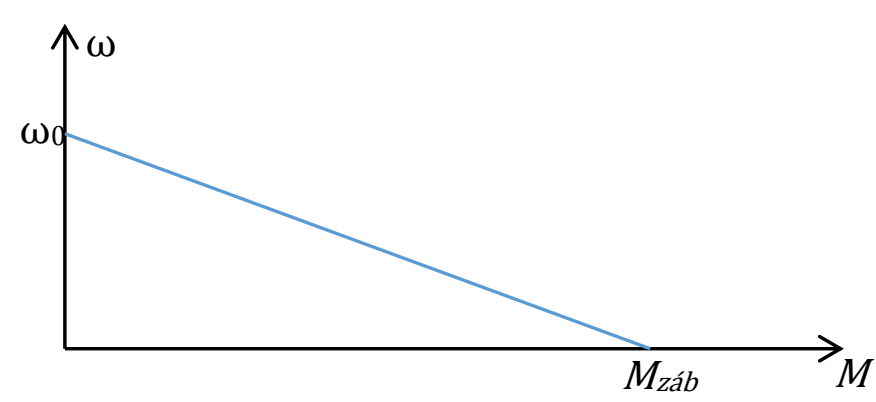

Fig. 6 Static torque characteristics of DC motor with permanent magnet

\section{THE ACCELEROMETER AND GYROSCOPE DATA PROCESSING}

The most important task of the control of self-balancing cart is the determination of the real inclination angle of the platform. Information about the movement and the angular position of the self-balancing cart can be easily obtained by the accelerometer and gyroscope implemented in IMU. The angle is not directly measurable, and it must be acquired indirectly using one of the three methods:

- Accelerometer - the inclination angle is computed as a projection of the vector of gravity into the horizontal axis of the sensor using atan2 function. However, the forward acceleration is also projected into the measured signal, and thus the angle can be computed very incorrectly.

- Gyrosensor - the angle is obtained as the integration of the measured angular velocity. The problem is the drift of the gyro data.

- Combination of both, accelerometer and gyrosensor - is the practical, most-widely used option [1].

There are two main problems when combining both above-mentioned sensors. The accelerometer measures true values when the acceleration is continuous; however, when vibrations occur, the returned values are laden with error. The gyroscope data has tendency to drift owing to the integration over time. Values did not return to zero when the system went back to its original position [1]. Solution to that problem is to merge the signals returned by the sensors by: 
- Kalman filter - the best filter to be used. From a theoretical point of view, it is the one that minimizes the errors from the true signal value. The filter needs to be able to calculate the coefficients of the matrices, the process-based error, measurement error, etc. Firstly, it is very difficult to understand, and secondly, it is hard to implement on low-cost hardware.

- First-order complementary filter - this is easy used filter. The filter combines the readings from the accelerometer and the gyroscope to get good usable results. This filter is used in control algorithm of self-balancing cart.

- Second-order complementary filter - the principle of the filter is the same, but the algorithm is more complicated as for first-order filter.

As follows from test results [6], the complementary filter can substitute the Kalman filter. From the theoretical point of view, the Kalman filter is the best one for such applications, but its complexity is too high for a hobbistic robot builders and bachelor degree students. The complementary filter is easier to use and fast faster and easier to use.

First-order complementary filter merges high-pass and low-pass filters simultaneously. The low-pass filter filters high frequency signals (such as vibrations in the accelerometer signal), while high-pass filter filters low frequency signal (such as the drift of the gyroscope), as shown in Fig. 7 [7]. To obtain the tilt angle by the accelerometer, we had to determine the position of the gravity vector, always accessible on the accelerometer. We could obtain the tilt angle from the gyroscope data by integrating the angular velocity over time.

The used MPU-6050 contains a digital motion processor (DMP) which can perform the data fusion on the IMU chip. The details of how the DMP performs the calculations have not been published. Comparison of DMP and complementary filter indicates that, for pitch (rotation about the $\mathrm{Y}$-axis) and roll (rotation about the $\mathrm{X}$-axis), the calculations are fairly close. The DMP algorithm is able to calculate yaw (rotation about the Z-axis), while the complementary filter cannot. However, it is possible that a correct implementation of the complementary filter might eliminate some of the errors.

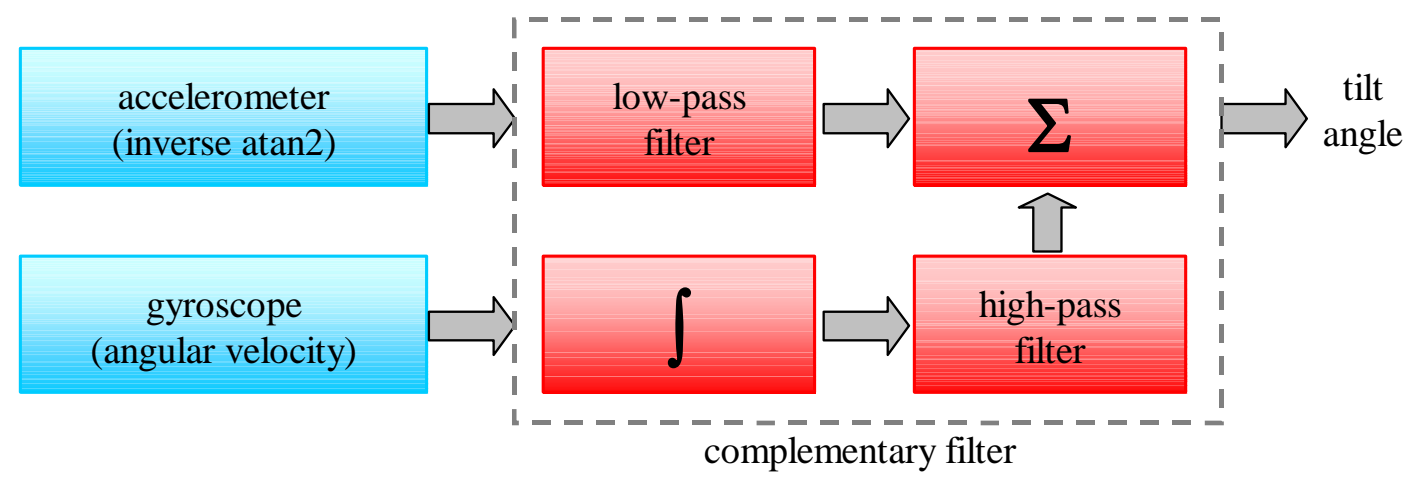

Fig. 7 Idea of the complementary filter

Implementation of complementary filter algorithm for the self-balancing cart takes the form

$$
\text { filtered } \theta=\alpha\left(\text { previous } \theta+\text { gyroAngVelo } Y^{*} d t\right)+(1-\alpha)(\text { accAngle }),
$$

where $\alpha=\tau /(\tau+d t)$ is the filter coefficient, $\tau$ is the time constant of the filter and $d t$ is the loop time period which depends on real implementation. In the algorithm of complementary filter, coefficient $\alpha=0.98$ was used. The data of gyro is a significant input to the filter, and the accelerometer data is just used for drift compensation. Orientation of the IMU sensor axes is shown in Fig. 9. To calculate accAngle, the following relationship was applied 


$$
\text { accAngle }=a c c X / \sqrt{a c c X^{2}+a c c Z^{2}} .
$$

To ensure the correct values of tilt angle and angular speed, the calibration of the IMU sensor (adjustment of gyro and accelerometer offsets) before the first use must be performed.

\section{PID CONTROL}

PID is used to control the motors of the self-balancing cart according to the rapid changes in tilt angle. The basic algorithm of PID control is well known (Fig. 5). In the self-balancing cart, the reference angle can be calculated by positioning the robot in the upright position, and therefore the feedback error value can be calculated as

$$
e(t)=\text { filtered } \theta-\text { SetPoint },
$$

where SetPoint is the reference tilt angle in which the cart is in a stable position. The value of SetPoint is related on the mass distribution in the system.

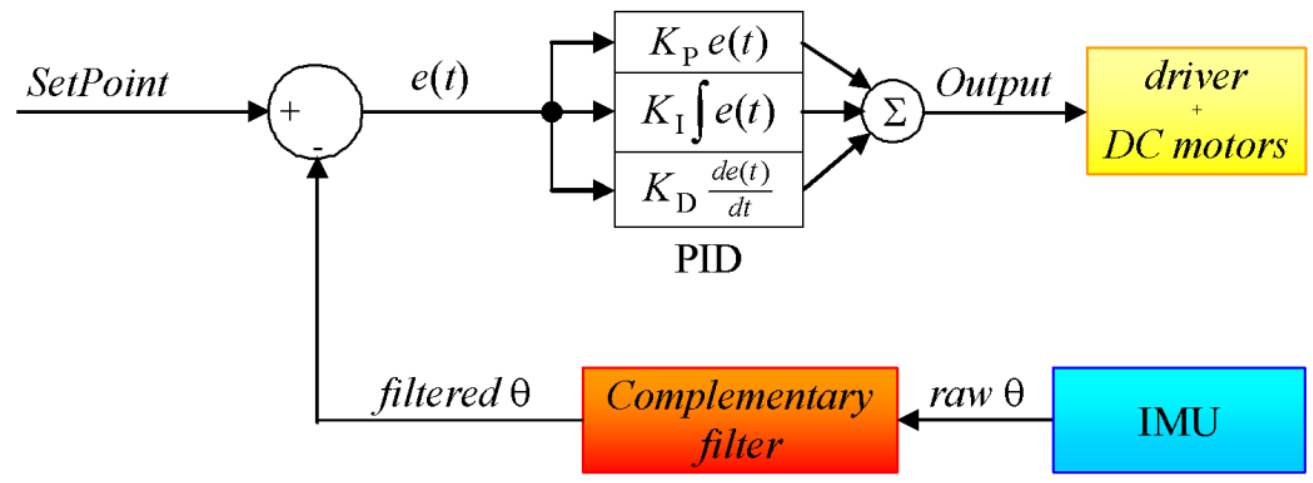

Fig. 8 Scheme of PID control system

Stabilization of the system required continuously taking the correct tilt feedback to provide the needful DC motors control according to the PID algorithm [9]. Throughout practical experiments, manual PID tuning was used to obtain the desired set of control term values. The Ziegler-Nichols tuning procedure gave an acceptable set of gain parameters at which the output of the control loop is stable. For real model of self-balance cart, value set $\left(K_{\mathrm{P}}=52, K_{\mathrm{I}}=75\right.$, $K_{\mathrm{D}}=0.3$ ) looks as optimal. In control algorithm of the model PID regulator, output is processed into the variable of Output, the value of which controls the operation of DC motors driver according to the conditions

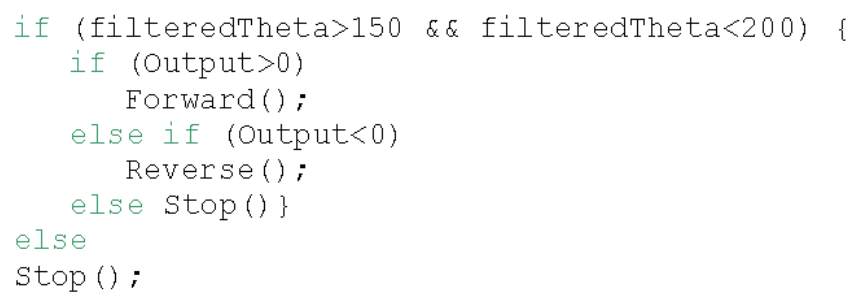

where the first condition (filteredTheta range) served to switch-off the motors in case of a complete loss of the vehicle stability. 


\section{MECHANICAL AND ELECTRICAL DESIGN}

The assembly of the self-balancing two-wheel cart is shown in Fig. 6. Mechanical part is made of aluminium profile and electric isolated rods. The control circuit contains Arduino Uno as the vehicle controller, IMU MPU-6050 as the angular accelerometer and gyrosensor, L298N as a driver controller, and two DC motors with gearbox and wheels. The mass, dimensional and computational parameters of the real model are summarized in Table 2.
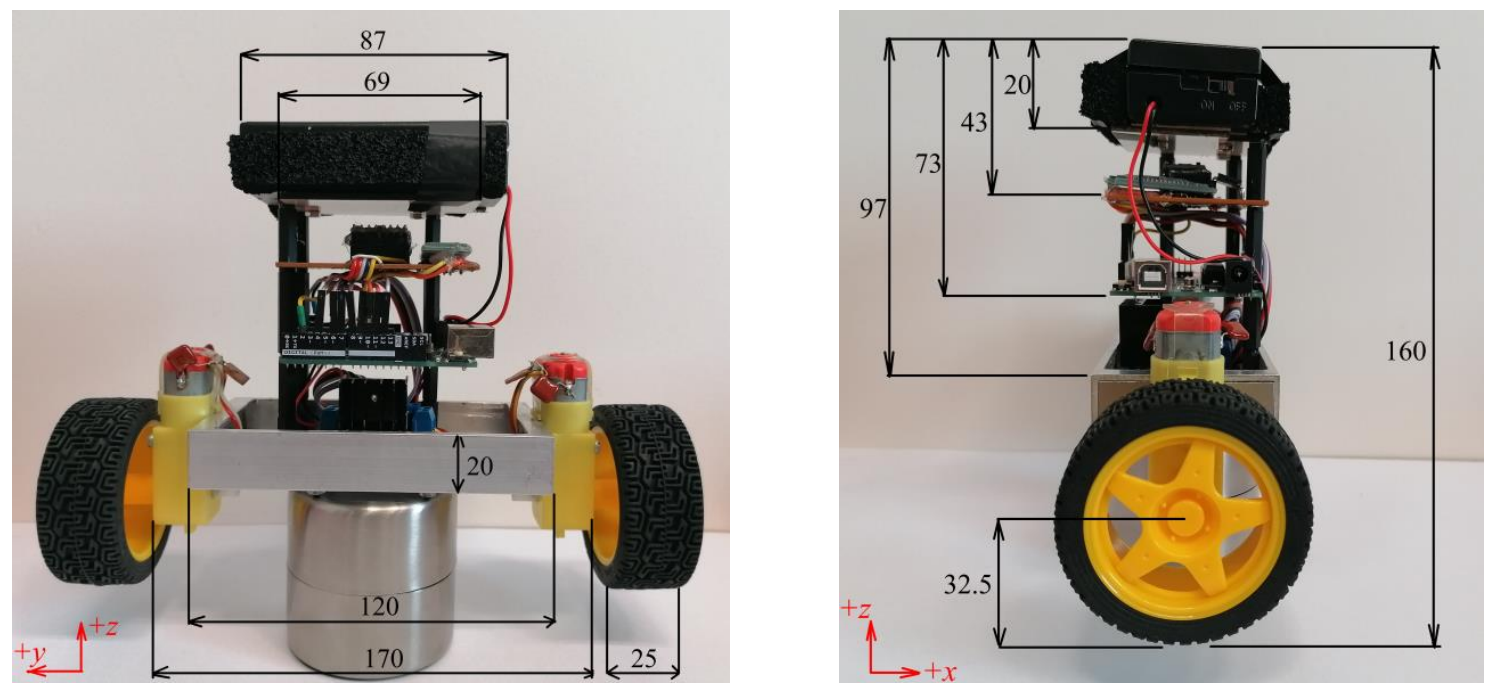

Fig. 9 Design and dimensions of two-wheel self-balancing cart model version

\begin{tabular}{ll}
\hline Table 2 Parameters of the real model & \\
\hline weight of chassis part & $m_{C}=0.454 \mathrm{~kg}$ \\
weight of one wheel & $m_{W}=0.0345 \mathrm{~kg}$ \\
moment of inertia of chassis part & $I_{C}=2.894 \times 10^{-4} \mathrm{~kg} \mathrm{~m}^{2}$ \\
moment of inertia of one wheel & $I_{W}=1.822 \times 10^{-5} \mathrm{~kg} \mathrm{~m}^{2}$ \\
dimensions of chassis part $(b \times h \times v)$ & $(170 \times 55 \times 127) \mathrm{mm}$ \\
wheel diameter (radius) & $D=65 \mathrm{~mm}(R=32.5 \mathrm{~mm})$ \\
distance between wheel axis and center of mass & $L_{\mathrm{T}}=38 \mathrm{~mm}$ \\
of the chassis part & $g=9.81 \mathrm{~m} \mathrm{~s}^{-2}$ \\
gravity & \\
\hline
\end{tabular}

Calculation limit of the cart tilt angle was set to $\theta_{\max }=20^{\circ}$, and angular speed $n=200$ RPM. The minimal torque $M_{\min }$ and power $P_{\min }$ of both DC motors are then given as

$$
M_{\text {min }}=m_{C} g L_{\mathrm{T}} \sin \theta_{\text {max }}=0.06039 \mathrm{Nm} \quad \text { and } \quad P_{\min }=\frac{2 \pi M_{\min } n}{60 \eta}=3.16 \mathrm{~W}
$$

at the considered efficiency of $80 \%(\eta=0.8)$. Based on the calculated values, DC motor 1B481416LSD6 was chosen with the following parameters: supply voltage 3 - $8 \mathrm{~V}$, motor current 70 - $250 \mathrm{~mA}$, gear ratio $i=1: 48$, torque $0.015-0.085 \mathrm{Nm}$ and rotational speed $110-250 \mathrm{RPM}$. Fig. 10 shows the electrical circuit used in the project. 


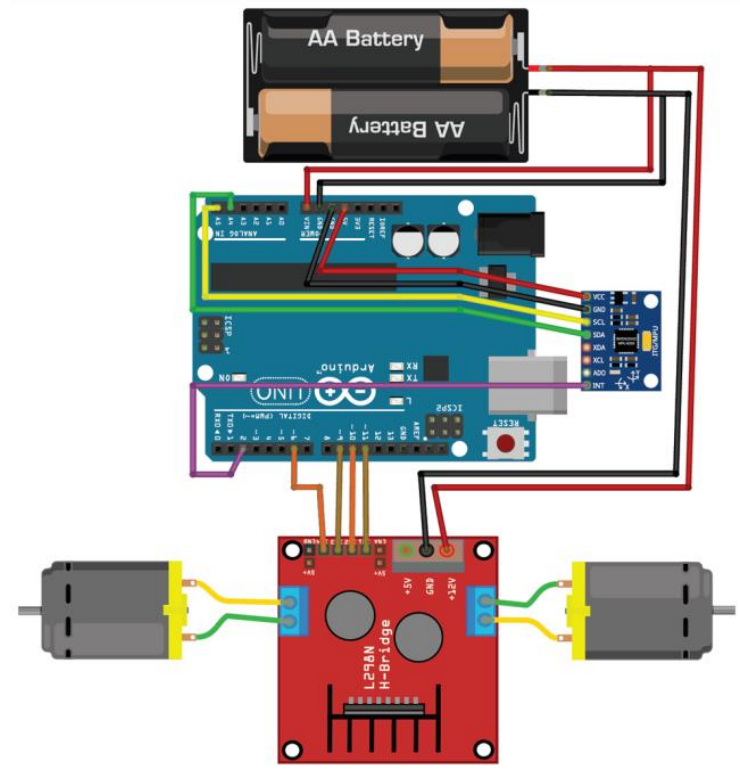

Fig. 10 Connection of DC motor driver and IMU to Arduino Uno

The implemented balance control algorithm functionality was verified on the made model based on the Arduino embedded development board. As shown in Fig. 11, variance of inclination angle around the stable position of the cart was in the range of $\pm 5^{\circ}$ approximately. Good functionality of the control algorithm is shown in the photo sequence in Fig. 12.

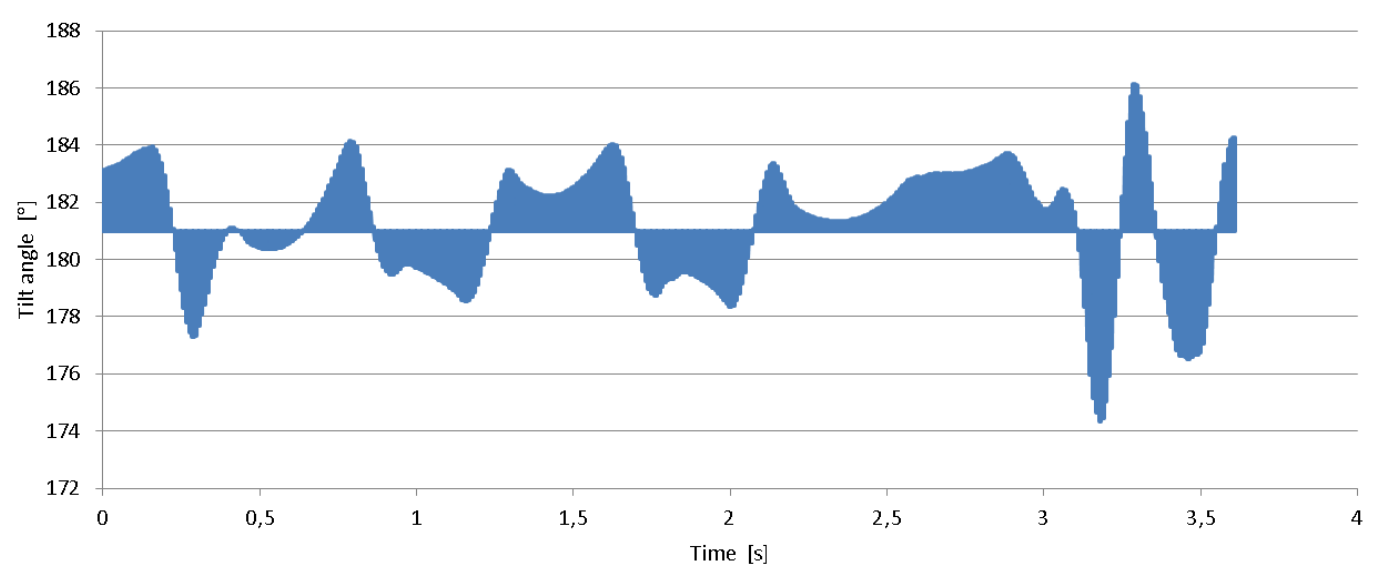

Fig. 11 Example of tilt angle variation over the time during the real model test

\section{Summary}

The purpose of research described in this paper was to propose a simple model and control system of inverted pendulum applicable in teaching process. The final implemented algorithm is very simple, consisting of the function to capture data from IMU, a complementary filter and PID controller of the cart motion. Structure of control algorithm allows to test influence on stability of self-balancing cart by changing filter types, their constants and the PID controller parameters. Currently, the robot can move forward and backward in the inclined plane, but it cannot turn left or right. The model successfully demonstrated potential for academic use. The modular design allows to connect additional sensors and accessories (distance sensor, communication module). In the future, the model will be complemented by remote control and the ability to change the direction of movement. 


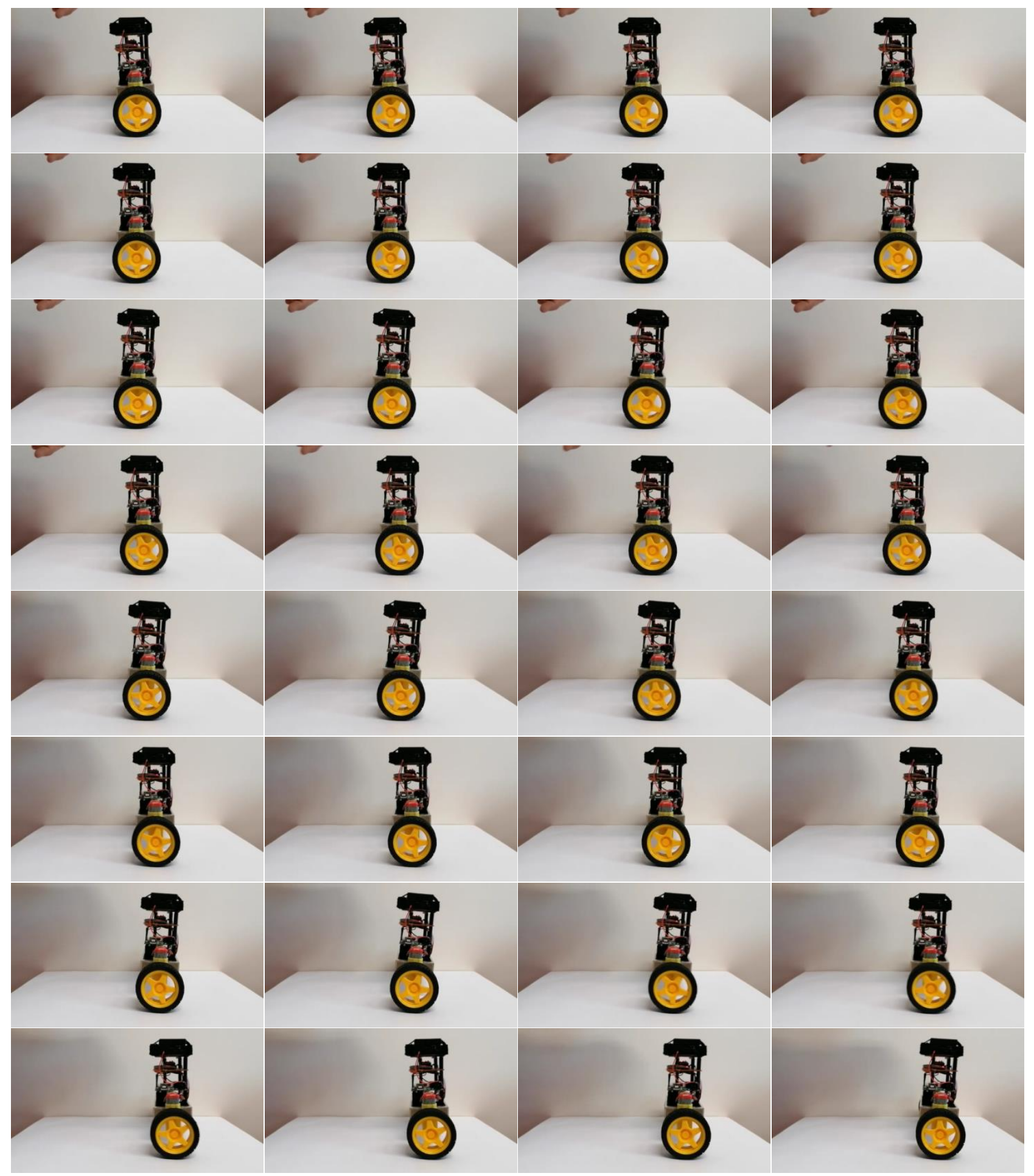

Fig. 12 Results of the stability test of two-wheel self-balancing cart

\section{Acknowledgement}

This project was supported by the Scientific Grant Agency of the Slovak Republic KEGA under the grant No. 009STU-4/2021. 


\section{References}

[1] COLTON, S. The balance filter - A simple solution for integrating accelerometer and gyroscope measurements for a balancing problem, Information on http://www.ee.ic.ac.uk/pcheung/teaching/de2_ee/complementary filter.pdf

[2] GONG, Y., WU, X., MA. H. 2015. Research on control strategy of two-wheeled self-balancing robot. In: 2015 International Conference on Computer Science and Mechanical Automation (CSMA), pp. 281-284.

[3] JUNFENG, W., WANYIN, Z. 2011. Research on control method of two-wheeled self-balancing robot. In 2011 Fourth International Conference on Intelligent Computation Technology and Automation, Volume 1, pp. 476-479.

[4] BABAZADEH, R., KHIABANI, A.G., AZMIB, H. 2015. Nonlinear modelling and optimal output control of two wheeled balancing transporter. J. of Computer \& Robotics., 8(2), 1-7.

[5] JAYAKODY, D.P.V.J., SUCHARITHARATHNA, K.P.G.C. 2019. Control unit for a two-wheel self-balancing robot. Global J. of Researches in Engineering. 19(1).

[6] WALTER, T., HIGGINS, J. R. 1975. A comparison of complementary and Kalman filtering, IEEE Transactions on Aerospace and Electronic Systems, 11(3).

[7] HIGGINS, W. T. 2015. A comparison of complementary and Kalman filtering. In: 10th Asian Control Conference (ASCC), pp. 1-6.

[8] DING, Y., GAFFORD, J., KUNIO, M. Modelling, simulation and fabrication of a balancing robot. Information on https://pdfs.semanticscholar.org/2fd5/119f144c38f599fe66715aead1ea717ee3cd.pdf

[9] Y. Y. LIM, CH. L. HOO, Y. M. F. 2018. Wong, Stabilising an Inverted Pendulum with PID Controller. In: MATEC Web of Conferences, 152.

[10] CAO, J. R., HUANG, C. P. and HUNG, J. C. 2016. Stabilizing controller design using fuzzy t-s model on two wheeled self-balancing vehicle. In: 2016 International Conference on Advanced Materials for Science and Engineering (ICAMSE), pp. 520-523.

\section{ORCID}

$\begin{array}{ll}\text { Rastislav Ďuriš } & 0000-0002-5984-8730 \\ \text { Eva Labašová } & 0000-0001-9055-1233 \\ \text { Jarmila Oravcová } & 0000-0002-2414-8064\end{array}$

